\title{
Adult age-dependent differences in resting-state connectivity within and between visual-attention and sensorimotor networks
}

\section{Christian Roski ${ }^{1}$, Svenja Caspers ${ }^{1 *}$, Robert Langner ${ }^{1,2}$, Angela R. Laird $^{3,4}$, Peter T. Fox ${ }^{3}$, Karl Zilles ${ }^{1,5,6}$, Katrin Amunts ${ }^{1,7}$ and Simon B. Eickhoff ${ }^{1,2}$}

${ }^{1}$ Research Center Jülich, Institute of Neuroscience and Medicine (INM-1, INM-2), Jülich, Germany

2 Institute of Clinical Neuroscience and Medical Psychology, Heinrich-Heine-University, Düsseldorf, Germany

${ }^{3}$ Research Imaging Institute, University of Texas Health Science Center, San Antonio, TX, USA

${ }^{4}$ Department of Physics, Florida International University, Miami, FL, USA

${ }^{5}$ Department of Psychiatry, Psychotherapy, and Psychosomatics, RWTH Aachen University, Aachen, Germany

6 JARA-BRAIN, Jülich-Aachen Research Alliance, Jülich/Aachen, Germany

${ }^{7}$ C. and O. Vogt Institute for Brain Research, Heinrich-Heine-University Düsseldorf, Düsseldorf, Germany

\section{Edited by:}

Emil C. Toescu, Birmingham

University, UK

\section{Reviewed by:}

Richard Camicioli, McGill University, Canada

Carlos Beas-Zárate, Universidad de Guadalajara, Mexico

*Correspondence:

Svenja Caspers, Research Center Jülich, Institute of Neuroscience and

Medicine, INM-1, Leo-Brandt Str. 1,

Jülich 52425, Germany

e-mail: s.caspers@fz-juelich.de
Healthy aging is accompanied by structural and functional changes in the brain, among which a loss of neural specificity (i.e., dedifferentiation) is one of the most consistent findings. Little is known, however, about changes in interregional integration underlying a dedifferentiation across different functional systems. In a large sample $(n=399)$ of healthy adults aged from 18 to 85 years, we analyzed age-dependent differences in resting-state (RS) (task-independent) functional connectivity (FC) of a set of brain regions derived from a previous $\mathrm{fMRI}$ study. In that study, these regions had shown an age-related loss of activation specificity in visual-attention (superior parietal area 7A and dorsal premotor cortex) or sensorimotor (area OP4 of the parietal operculum) tasks. In addition to these dedifferentiated regions, the FC analysis of the present study included "task-general" regions associated with both attention and sensorimotor systems (rostral supplementary motor area and bilateral anterior insula) as defined via meta-analytical co-activation mapping. Within this network, we observed both selective increases and decreases in RS-FC with age. In line with regional activation changes reported previously, we found diminished anti-correlated FC for inter-system connections (i.e., between sensorimotor-related and visual attention-related regions). Our analysis also revealed reduced FC between system-specific and task-general regions, which might reflect age-related deficits in top-down control possibly leading to dedifferentiation of task-specific brain activity. Together, our results underpin the notion that RS-FC changes concur with regional activity changes in the healthy aging brain, presumably contributing jointly to age-related behavioral changes.

Keywords: aging, fMRI, resting state, functional connectivity, MACM, functional systems

\section{INTRODUCTION}

Effective information processing depends on the integrity of communication between the different nodes of functional brain systems. Through normal aging, substantial changes within and between brain networks occur (for review see Eyler et al., 2011; Grady, 2012). For example, it has been shown, that older adults exhibit lower connectivity within task-relevant networks and greater connectivity outside the task-relevant networks (Daselaar et al., 2006; Dennis et al., 2008; St Jacques et al., 2009). Moreover, changes of regional brain activity were repeatedly observed in older adults (cf. Eyler et al., 2011; Grady, 2012). These neural changes are in line with behavioral studies reporting increased associations between sensory, sensorimotor, and cognitive functions in elderly participants (Lindenberger and Baltes, 1994; Baltes and Lindenberger, 1997; Schaefer et al., 2006).
In a previous functional magnetic resonance imaging (fMRI) study, we investigated functional activation patterns in the sensorimotor and the visual attention systems in a large sample of healthy subjects between 20 and 70 years of age (Roski et al., 2013). When testing for age-related effects across both functional domains (visual attention and sensorimotor control), we found reduced activation in several task-relevant regions as well as increased activation in regions that were less activated in younger adults. This effect holds substantial similarity to the repeatedly observed age-related process of dedifferentiation, i.e., a loss of neural specificity within distinct functional systems (for review see Reuter-Lorenz and Park, 2010). Little is known, however, about the aberrations in neural networks, i.e., interregional integration, underlying a dedifferentiation across different functional systems as observed in that previous fMRI 
study. This raises the question to which extent the interaction within and between functional systems becomes less differentiated as a consequence of healthy aging. In this context it should be noted that age-related changes in the interplay between visual attention processes and the sensorimotor system might provide additional insights into the physiological changes across multiple brain systems with healthy aging. In addition to providing a complementary, network-based perspective on age-related dedifferentiation across systems, improved knowledge of age-related changes in functional integration may also provide an important background for the assessment of network pathology caused by neurodegenerative disorders. For example, it is assumed that the cognitive deficits in Alzheimer's disease may be attributed to the disease's severe effects on functional networks marked by a profound disruption of functional connectivity (FC) within and across brain networks (for review see Delbeuck et al., 2003). In this context, however, it remains an open question whether similar age-related FC changes in connectivity between/within the sensorimotor and visualattention systems may also be found in healthy aging, as suggested by the previously observed, dedifferentiated, recruitment pattern.

These considerations promoted the current analysis of agerelated changes in functional integration between regions of the visual-attention and sensorimotor systems that were previously shown to feature reduced functional specificity with increasing age. Our aim was thus to address the changes in network interactions underlying the observed dedifferentiation across functional domains. This goal was pursued by investigating age-related alteration of task-independent, i.e., resting-state (RS), FC between regions of the visual-attention or sensorimotor systems (functional seeds) that show a dedifferentiated recruitment pattern with increasing age. In this context it should be noted that temporally correlated brain activity in spatially distinct regions (i.e., FC) may not only arise from direct interaction (Eickhoff and Grefkes, 2011). Rather, FC, particularly between different functional systems, may also be mediated by task-general regions interacting with regions from either system. The inclusion of these regions (in addition to the functionally defined seed regions) in the FC analysis should hence offer insights into the mechanisms underlying the change of network interactions between the brain systems in the elderly.

In the present study, we investigated changes of RS-FC between functional seeds that showed an age-related dedifferentiated recruitment pattern and task-general regions that are functionally related to them. The functional seeds were defined based on results from a previous fMRI study (Roski et al., 2013), whereas the task-general brain regions were identified via metaanalytic connectivity modeling (MACM; Eickhoff et al., 2010; Robinson et al., 2010). Once these task-general regions were defined, a two-step RS-FC network analysis was performed. In the first step, the task-independent connectivity pattern of all regions was analyzed in a large adult sample $(n=399)$. In the second step, age-related changes in task-independent FC for this set of seed regions were analyzed in the same sample. This approach of examining the RS-FC including task-general regions in a large sample of healthy subjects should provide insights into age-related changes in the functional coupling between brain regions involved in sensorimotor and visual attention processing.

\section{MATERIALS AND METHODS}

The initial seed regions for the current connectivity analysis were provided by regions that showed a less differentiated neural activity pattern across the sensorimotor and the visual attention systems with age, i.e., an age-by-task interaction (Roski et al., 2013). As mentioned above, additional task-general regions (i.e., regions that consistently interact with each of these seed regions) were defined using MACM. The ensuing set of brain regions (seed regions from the fMRI study and task-general regions from the MACM analysis) were analyzed in a twostep RS-FC network analysis to unveil (i) the task-independent inter-regional FC within the combined set of brain regions, and (ii) to analyze age-related changes of FC within this network. All specified analyses are described in detail in the following sections.

\section{DEFINITION OF SEED REGIONS \\ Seed regions based on $\mathrm{AMRI}$}

Seed regions were derived from a previous fMRI study on age-related changes in neural correlates of sensorimotor and visual attention processing (Roski et al., 2013). For the present study, only regions that showed an age-by-task interaction were included (Figure 1): bilateral area OP4 of the parietal operculum (Eickhoff et al., 2006a,b) showed a decrease in activation during a motor task (finger tapping) but an increase in activation during a visual attention task (target letter counting) in elderly participants. In contrast, bilateral superior parietal area 7A (Scheperjans et al., 2008a,b) and the rostral part of the dorsal premotor cortex (dPMC; cf. Brown et al., 2004; Ford et al., 2005; Amiez et al., 2006) showed the opposite pattern: an age-related decrease in activation during the visual attention task and an increase in activation during the motor task.

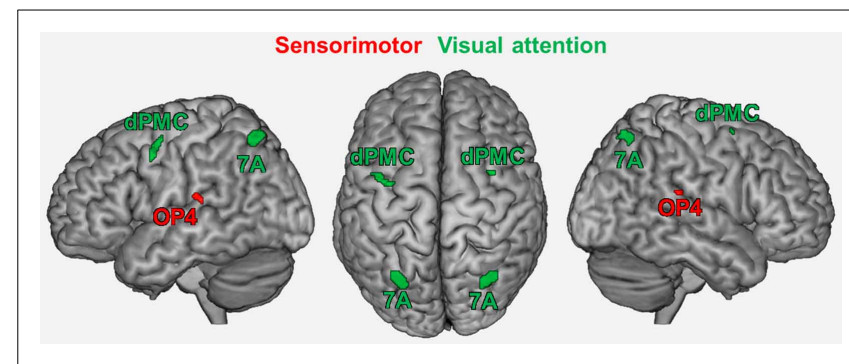

FIGURE 1 | Regions showing an age by task interaction as reported in a previous study (Roski et al., 2013). These regions represent the fMRI-based seed regions for the present study. Green color denotes regions associated with visual attention; red color denotes regions associated with sensorimotor processing. 7A, superior parietal lobule area 7A (cytoarchitectonically defined; Scheperjans et al., 2008a,b); dPMC, dorsal premotor cortex; OP4, area OP4 of the parietal operculum (cytoarchitectonically defined; Eickhoff et al., 2006a,b). 


\section{Task-general regions}

Besides these functionally defined seeds, we also included taskgeneral regions in the current analysis. We considered those regions to be task-general since, across tasks, they showed a consistent functional relation to each of the regions derived from the fMRI study. To identify these regions, we first mapped the taskdependent co-activation pattern for each of the above-mentioned seed regions using MACM. Subsequently, a conjunction analysis across the resulting co-activation maps using a minimum statistic approach revealed those regions that were functionally related to all fMRI-based seeds.

Here we used the BrainMap database (Laird et al., 2009, 2011; www.brainmap.org) to assess the co-activation pattern of each seed (Eickhoff et al., 2010), considering all experiments which reported stereotaxic coordinates from normal mapping studies (no interventions and no group comparisons) in healthy subjects using either fMRI or positron emission tomography (PET). These inclusion criteria yielded (at the time of analysis) $\sim 7200$ functional neuroimaging experiments. For each individual seed region (left/right OP4, 7A, DPMC) we proceeded as follows: first, we identified the 100 experiments in BrainMap that reported activation closest to it (see Table 1). Then we tested for convergence across (all) foci reported in these experiments using the revised version (Eickhoff et al., 2009) of the activation likelihood estimation (ALE) approach. Using random-effects inference, the ALE maps reflecting the convergence of co-activations with each seed region, were thresholded at $p<0.05$ (cluster-level family wise error corrected; cluster-forming threshold: $p<0.001$ at voxel level) and converted to Z-scores for visualization (see supplementary material Figure S1). As experiments were selected by activation close to the seed, highest convergence will be observed in the seed region ( $\mathrm{cf}$. Table $\mathbf{1}$ ). Significant convergence in other brain regions in turn indicates consistent co-activation over experiments and, hence, FC with the seed (Jakobs et al., 2012).

To identify task-general regions for the subsequent RS-FC analysis, i.e., regions that were significantly co-activated with all seeds, we performed a conjunction analysis across the respective MACM results using the conservative minimum statistic (Nichols et al., 2005). This approach showed that the bilateral anterior insular cortex (AIC; MNI: -32, 20, 6; 36, 18, 4) was consistently co-activated with all seed regions (Figure 2 ). Also a region within the rostroventral supplementary motor cortex (SMAr; 2,

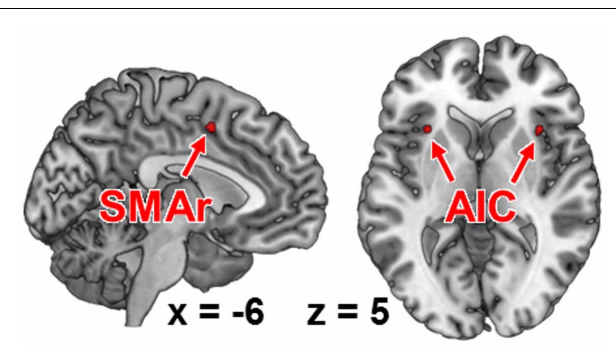

FIGURE 2 | Functional intersection of all co-activation maps of each fMRI-based seed region, as revealed by meta-analytic connectivity modeling. These regions represent those brain areas that were functionally connected to all of our seed regions (i.e., task-general regions). AIC, anterior insular cortex; SMAr, rostroventral supplementary motor area.
$0,56)$, rostrally bordering the pre-SMA and ventrally bordering the middle cingulate cortex (Palomero-Gallagher et al., 2008, 2009; Hoffstaedter et al., 2012), was consistently co-activated with each seed region. Both regions were hence defined to hold a task-general function across sensorimotor and visual attention processes.

\section{Combined set of seed regions-fMRI-based seed regions and task-general regions}

The fMRI-based seed regions (visual attention and sensorimotor) and the MACM-derived task-general seed regions conjointly represented the combined set of our seed regions, comprising sensorimotor area OP4 and attention-related $\mathrm{DPMC}$ and area 7A, as fMRI-based seed regions, as well as SMAr and AIC as task-general regions (Figure 3).

\section{RESTING-STATE ANALYSIS}

An RS-FC network analysis was implemented to analyze the taskindependent FC between these seed regions and its change with

Table 1 | Location of seed regions and maximum distance to relevant foci within BrainMap.

\begin{tabular}{lll}
\hline $\begin{array}{l}\text { Seed } \\
\text { region }\end{array}$ & $\begin{array}{l}\text { Peak coordinate } \\
\text { (MNI: } \mathbf{x}, \mathbf{y}, \mathbf{z})\end{array}$ & $\begin{array}{l}\text { Maximum } \\
\text { distance }(\mathbf{m m})\end{array}$ \\
\hline 7A left & $-29,-66,57$ & 6.9 \\
7A right & $32,-66,56$ & 7.1 \\
OP4 left & $-44,-17,12$ & 7.3 \\
OP4 right & $50,-14,11$ & 7.4 \\
dPMC left & $-41,2,50$ & 5.7 \\
dPMC right & $35,6,60$ & 7.8 \\
\hline
\end{tabular}

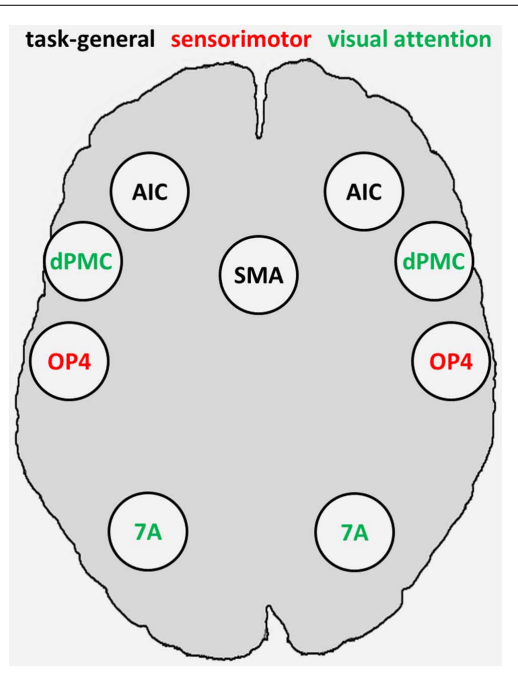

FIGURE 3 | Combined set of seed regions (schematic), comprising fMRI-based seed regions (visual attention and sensorimotor) derived from Roski et al. (2013) and task-general regions derived from meta-analytic connectivity modeling. 7A, superior parietal lobule area 7A; AIC, anterior insular cortex; dPMC, dorsal premotor cortex; OP4, area OP4 of the parietal operculum; SMAr, rostroventral supplementary motor area. 
age for all possible connections between the combined set of seed regions (fMRI-based and task-general).

\section{Sample}

Inter-regional RS-FC for the combined set of seed regions was assessed by using RS fMRI data from 399 healthy volunteers, aged $18-85$ years $($ Mean $=41.8, S D=16.8$, Median $=41, I Q R=29)$. All participants ( $46 \%$ female) were without any record of neurological or psychiatric disorders and gave their written informed consent to participate in the study. The data were contributed by four sites (see Table 2). Joint (re-)analysis of the data was approved by the local ethics committee of the Heinrich Heine University Düsseldorf.

\section{Imaging and pre-processing}

During scanning participants were instructed to let their mind wander but not to fall asleep which was confirmed by postscan debriefing. For each subject the RS EPI images were acquired using blood-oxygen-level-dependent (BOLD) contrast (cf. Table 1). Image acquisition was preceded by dummy images allowing for magnetic field saturation which were discharged prior to further processing using SPM8 (www.fil.ion.ucl.ac.uk/ $\mathrm{spm}$ ). The EPI images were first corrected for head movement by affine registration using a two-pass procedure. For normalization the mean EPI images were segmented into gray matter, white matter and cerebrospinal fluid using the "unified segmentation" approach (Ashburner and Friston, 2005). The resulting parameters of a discrete cosine transform, which define the deformation field necessary to move subject data into MNI space, were then combined with the deformation field transforming between the latter and the MNI single-subject template. The ensuing deformation was subsequently applied to the individual EPI volumes which thereby were transformed into the MNI single-subject space and resampled at $1.5 \mathrm{~mm}$ isotropic voxel size. Finally, images were smoothed by a $5-\mathrm{mm}$ FWHM Gaussian to meet requirements of the general linear model and compensate for residual anatomical variations.

\section{Analysis}

FC analyses may be influenced by several confounds such as physiological processes, e.g., fluctuations related to cardiac and respiratory cycles and in particular motion-related effects (Bandettini and Bullmore, 2008; Fox et al., 2009). In order to reduce spurious correlations, variance that could be explained by the following nuisance variables was removed from each voxel's time series (Reetz et al., 2012; Zu Eulenburg et al., 2012; Satterthwaite et al., 2013): (i) the six motion parameters derived from the image realignment, (ii) the first derivatives of the six motion parameters, (iii) mean gray matter, white matter and cerebrospinal-fluid signal per time point as obtained by averaging across voxels attributed to the respective tissue class in the SPM8 segmentation. All nuisance variables entered the model as first and second order terms. Following confound removal data was band pass filtered preserving frequencies between 0.01 and $0.08 \mathrm{~Hz}$ (Biswal et al., 1995; Greicius et al., 2003; Fox and Raichle, 2007). The time course of each seed region was then extracted for each subject as the first eigenvariate of all gray-matter voxels located within $5 \mathrm{~mm}$ of the peak coordinate. For each subject the time-series data of all seed regions was then cross-correlated to quantify the degree of FC between the seed regions. The ensuing pair-wise correlation coefficients were subsequently transformed into Fisher's $\mathrm{Z}$ scores. Statistically significant connectivity was assessed via one-sample $t$-tests ( $p<0.05$, corrected for multiple comparisons using the false discovery rate). Subsequently, the same Fisher$\mathrm{Z}$ transformed correlation coefficients for each connection were rank-correlated with age to test for age-related changes in interregional coupling. The results of this correlation analysis were regarded significant if they passed a threshold of $p<0.05$, corrected for multiple comparisons using the false discovery rate. Finally, we analyzed the connectivity in the 100 youngest and 100 oldest participants for all connections showing significant age-related changes, to corroborate the correlational findings. Moreover, this analysis permits a more detailed analysis of each age-related change of RS-FC to test, e.g., if an age-related FC increase represents a significant change from negative to positive FC or from negative to absent FC. The former pattern would imply an inversion of the RS communication (i.e., an increased functional interplay for relevant regions), while the latter would imply a loss of communication between brain regions.

\section{RESULTS}

\section{RS-FC WITHIN AND BETWEEN FUNCTIONAL SYSTEMS}

We found significant RS-FC for several connections within the combined set of seed regions. Significant positive coupling was

Table 2 | Characteristics of the sample.

\begin{tabular}{|c|c|c|c|c|}
\hline Source site & $n$ & Mean age (range) & Sex: male (\%) & Measurement parameters ${ }^{a}$ \\
\hline RWTH University Hospital Aachen, Germany & 28 & $63.4(55-72)$ & 71 & $3 \mathrm{~T} / 270 / 2.2 / 30 / 90^{\circ} / 3.1 \times 3.1 \times 3.1 \mathrm{~mm}^{3}$ \\
\hline \multirow[t]{2}{*}{ Research Center Jülich, Germany } & 51 & $28.3(18-59$ & 57 & $3 \mathrm{~T} / 250 / 2.2 / 30 / 90^{\circ} / 3.1 \times 3.1 \times 3.1 \mathrm{~mm}^{3}$ \\
\hline & 100 & $45.1(21-71)$ & 48 & $3 \mathrm{~T} / 300 / 2.2 / 30 / 90^{\circ} / 3.1 \times 3.1 \times 3.1 \mathrm{~mm}^{3}$ \\
\hline
\end{tabular}

ICBM, International Consortium for Brain Mapping; NKI, Nathan S. Kline Institute.

a Measurement parameters: magnetic field strength of the scanner/number of acquired volumes/repetition time (in s)/echo time (in ms)/flip angle/voxel size.

${ }^{b}$ These data were selected from the datasets included in Biswal et al. (2010) and made publicly available via the 1000 Functional Connectomes Project. 


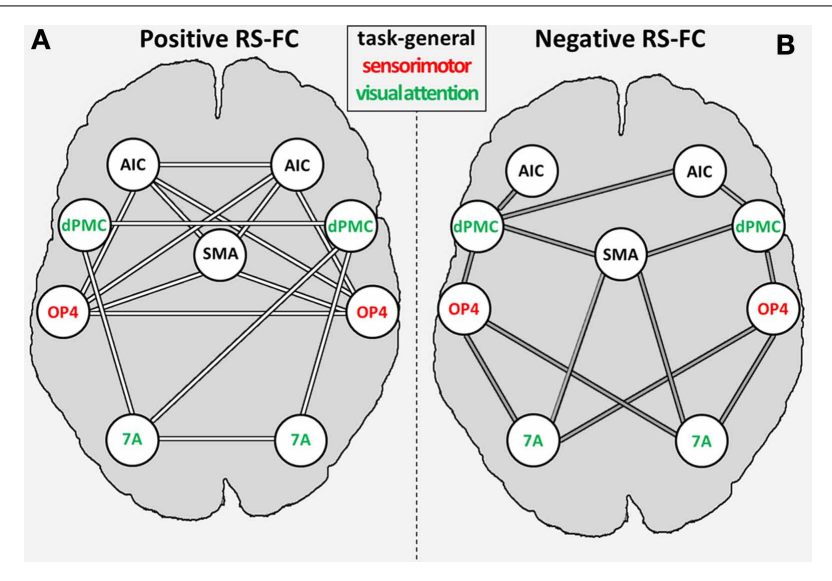

FIGURE 4 | General positive (A) and negative (B) inter-regional resting-state functional connectivity (RS-FC) for the combined set of seed regions. Regions labeled in green are associated with the visual attention system; regions labeled in red are associated with the sensorimotor system [as indicated by the previous fMRI study (OP4, dPMC, and 7A; Roski et al., 2013)]; regions labeled in gray have no functional preference (i.e., task-general regions). 7A, superior parietal lobule area 7A; $\mathrm{AIC}$, anterior insular cortex; dPMC, dorsal premotor cortex; OP4, area OP4 of the parietal operculum; SMAr, rostroventral supplementary motor cortex.

found for: (i) SMAr with bilateral OP4 and bilateral AIC; (ii) left AIC with bilateral OP4; (iii) right AIC with bilateral OP4 and right area 7A; (iv) bilateral dPMC each with ipsilateral area 7A; (v) right DPMC with left area 7A; (vi) interhemispheric connections between bilateral regions AIC, dPMC, OP4, and area 7A (see Figure 4A). Noticeably, positive coupling was thus predominately found between regions with similar functional preferences (in the fMRI study), i.e., intra-domain connections (Figure 4A).

Significant negative coupling (i.e., anti-correlations) was found for the following connections: (i) SMAr with bilateral area $7 \mathrm{~A}$ and right $\mathrm{APMC}$; (ii) bilateral OP4 to bilateral area 7A and dPMC; (iii) right AIC with bilateral dPMC and left AIC with left dPMC (see Figure 4B). Negative RS coupling was thus predominantly observable for inter-domain connections (Figure 4B), i.e., for regions with differing functional preferences in the original fMRI study.

\section{AGE-RELATED CHANGES OF RS-FC}

For several connections, significant age effects on RS-FC were found (Figure 5). Specifically, RS-FC between SMAr and bilateral $\mathrm{AIC}$ and $\mathrm{OP} 4$, respectively, decreased with age. Also, the interhemispheric connectivity between left and right area OP4 and left and right AIC decreased with age. In contrast, an age-related increase of RS-FC was found for the bilateral connection of the DPMC and area 7A to the SMAr. Furthermore, area 7A bilaterally showed increased RS-FC to ipsilateral area OP4, whereas the right area 7A additionally showed an age-related increase of FC to the contralateral OP4. The supplementary extreme-group analysis in which we directly compared the 100 youngest and 100 oldest participants yielded significant differences for all connections that showed a significant association between RS-FC and age (see Table 3), corroborating the age-related correlational findings.

\section{DISCUSSION}

The present study investigated age-related differences in taskindependent FC among a set of brain regions that was based on a previous fMRI study (Roski et al., 2013) to show a dedifferentiated recruitment pattern for visual-attention or sensorimotor demands (sensorimotor area OP4 and attention-related areas 7A and $\mathrm{dPMC}$ ). This set was supplemented by task-general regions showing significant co-activation with all functional seeds across a broad range of tasks as identified by MACM analyses (SMAr and bilateral AIC). Together, these regions were used as seed regions for a network RS-FC analysis. This analysis demonstrated that regions belonging to the same functional domain show positive FC with each other, whereas regions belonging to different functional domains are anti-correlated with each other. The analysis of age-related changes in the RS-FC within the network revealed selective alterations of inter-regional connections, highlighting potential neural correlates of common age-related behavioral changes.

\section{MAIN EFFECTS OF RESTING-STATE FUNCTIONAL CONNECTIVITY}

The RS-FC analysis showed significant positive or negative correlations between RS activity of several seeds. When contrasting the RS-FC pattern with the task-dependent connectivity pattern (MACM), some connections showed corresponding FC, whereas other connections showed no FC congruency (for a detailed discussion see supplementary material). Positive RS-FC was predominantly observed between brain regions associated with similar functional systems (Figure 5A), whereas negatively correlated RS-FC was predominantly observed between regions belonging to different functional systems (see Figure 5B). In other words, RS activity in regions with similar functional characteristics appears to be positively correlated, whereas RS activity in regions with different functional properties is negatively correlated. In summary, the (original) functional distinction of the combined set of seed regions was well corroborated by its RS-FC pattern. Our results are thus in line with previous studies showing temporal correlations across functionally related areas, thereby forming RS networks that mirror task-related functional systems (De Luca et al., 2006; Smith et al., 2009; Biswal et al., 2010).

In turn, we found that negative $\mathrm{FC}$ was predominantly found between regions from different domains. These results underline an intrinsic, functionally driven organization of the human brain and are supported by studies showing that regions with apparently opposing functionality are negatively correlated in their RS-FC (Greicius et al., 2003; Roskies et al., 2013; Schlegel et al., 2013).

\section{AGE-DEPENDENT DIFFERENCES IN RS-FC}

First of all, it is noteworthy that not all connections showed age-related changes, arguing against a general and unspecific age-related decline in the task-independent functional coupling between brain regions. Instead, it appears that changes across the lifespan occur selectively. Connections that showed age-related changes, however, mostly featured reductions of functional correlations, as both positive and negative RS-FC moved closer to zero with age. In other words, the task-independent functional correlation (i.e., negative or positive RS-FC) within and between 


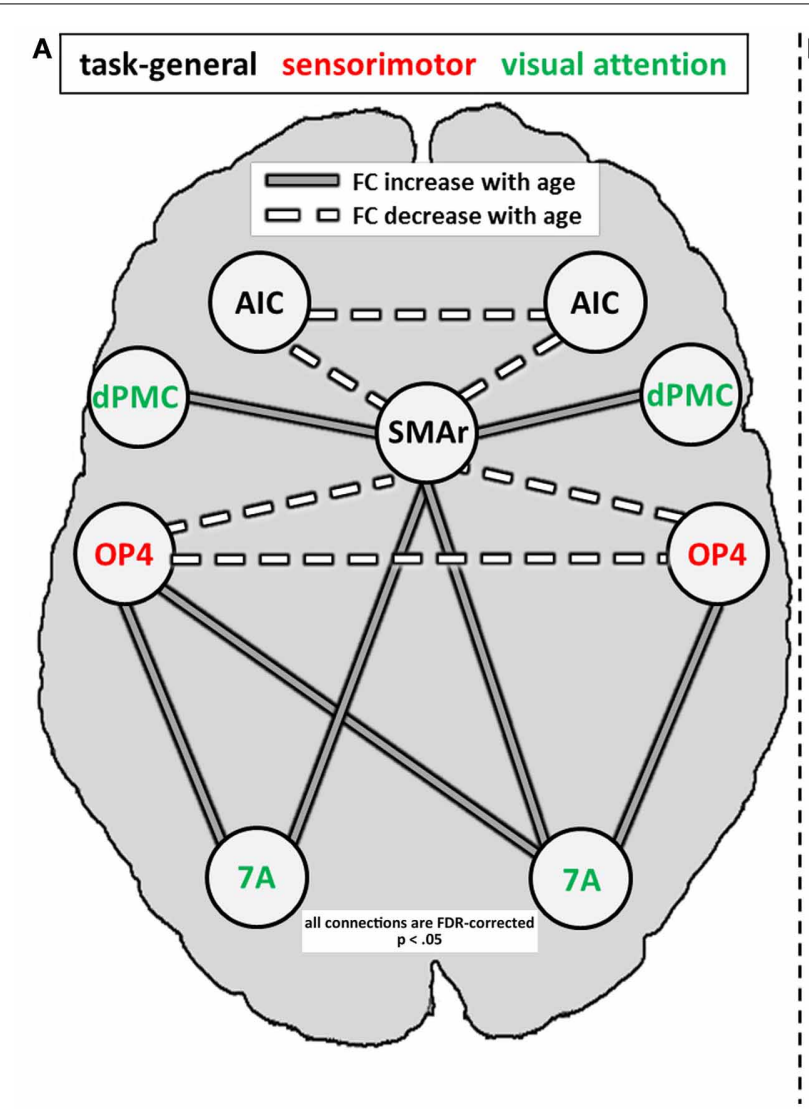

FIGURE 5 | (A) Alteration of resting-state functional connectivity (FC) with age (schematic). White lines reflect significant positive resting-state FC between regions (independent of age); gray lines reflect significant negative resting-state FC between regions (independent of age). Dotted lines reflect a significant decrease $[p<0.05$, corrected for multiple comparisons using the false discovery rate (FDR)] of FC with age; solid lines reflect a significant increase $(p<0.05$, FDR-corrected) of FC with

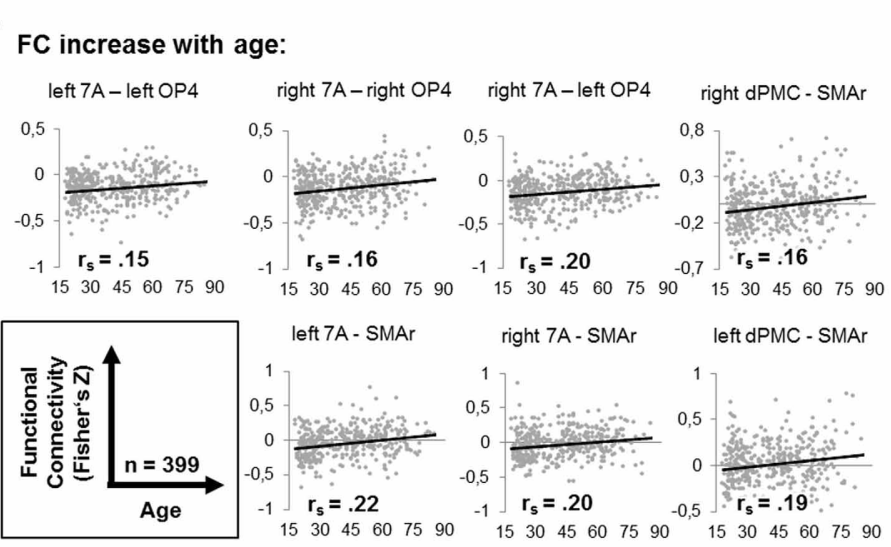

FC decrease with age:
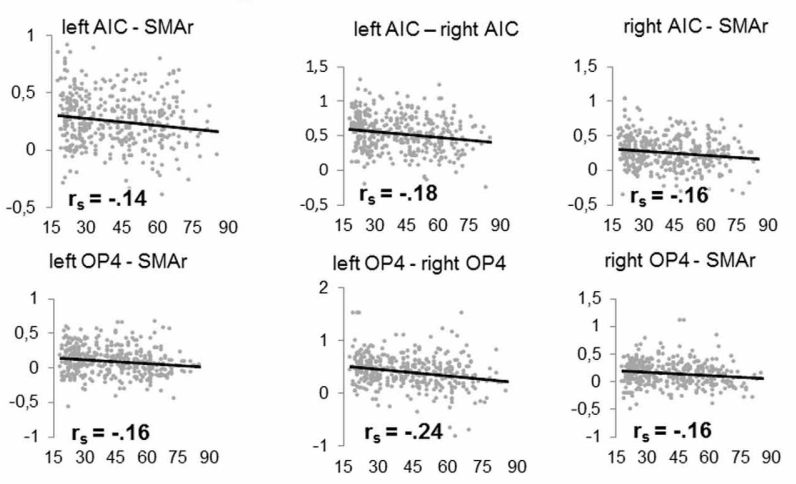

age. (B) Scatter plots for each age-affected interregional connection. Black line $=$ linear regression line; $r_{s}=$ Spearman correlation coefficient: a negative value reflects a decrease of $\mathrm{FC}$ with age, whereas a positive value reflects an increase of $\mathrm{FC}$ with age. $7 \mathrm{~A}$, superior parietal lobule area 7A; AIC, anterior insular cortex; dPMC, dorsal premotor cortex; OP4, area OP4 of the parietal operculum; SMAr, rostroventral supplementary motor cortex. the present networks appears to be diminished in older adults (see Figure 5B) making the network structure less distinct. According to the "disconnection" hypothesis proposed by O'sullivan et al. (2001), such functional "disruptions" in a network are associated with deteriorated white matter integrity and poorer cognitive performance across several functional domains. In line with this, a decreased FC within a distinct network, i.e., the default-mode network (Andrews-Hanna et al., 2007; Tomasi and Volkow, 2012), which is relevant for internally directed mental states including remembering, planning, and related cognitive functions (Greicius et al., 2003; Fransson, 2005; Buckner and Carroll, 2007) was demonstrated with age. In contrast to this, our findings indicate less RS communication between different task-related networks (i.e., visual-attention and sensorimotor networks). Nevertheless, we also found a few connections showing an increase in FC. The healthy aging brain is thus not only subject to functional decline but rather responds selectively to presumable structural or biochemical neuronal changes in the elderly. Again, this finding is particularly important in that it underlines that connectivity is not reduced per se, which may potentially be deemed to reflect systematic confounds. Rather, we found decreased (closer to zero), increased, or unchanged connections in our large sample.

\section{Age-dependent differences in RS-FC within the sensorimotor system}

The present results showed an age-related reduction of the taskindependent interhemispheric connection of the left and right area OP4. This region is assumed to play a role in sensorimotor integration processes, such as incorporating sensory feedback into motor actions (Rizzolatti and Wolpert, 2005; Halsband and Lange, 2006) and tactile object recognition and manipulation (Inoue et al., 2002; Wasaka et al., 2005). As a consequence of the decreased interhemispheric FC for this region, a diminished communication within this "sensorimotor feedback" system may develop in advanced age. A study on age-related behavioral slowing in sensorimotor tasks, suggesting a dysregulation of sensorimotor processing (Yordanova et al., 2004), corroborates this assumption. In line with this, increased age is associated with slower performance in speeded motor tasks (Salthouse, 2000). 
Table 3 | Age differences in intrinsic functional connectivity.

\begin{tabular}{lclc}
\hline Connection & Mean $\boldsymbol{r}_{\text {young }}$ & Mean $\boldsymbol{r}_{\text {old }}$ & $\begin{array}{l}\text { Correlation } \\
\text { with age }\left(\boldsymbol{r}_{\boldsymbol{s}}\right)\end{array}$ \\
\hline L 7A-L OP4 & $-0.164^{* * *}$ & $-0.087^{* * *}$ & 0.15 \\
L 7A-SMAr & $-0.111^{* * *}$ & -0.004 & 0.22 \\
R 7A-L OP4 & $-0.175^{* * *}$ & $-0.093^{* * *}$ & 0.20 \\
R 7A-R OP4 & $-0.151^{* * *}$ & $-0.071^{* * *}$ & 0.16 \\
R 7A-SMAr & $-0.077^{* *}$ & 0.019 & 0.20 \\
L AIC-R AIC & $0.515^{* *}$ & $0.445^{* * *}$ & -0.18 \\
L dPMC-SMAr & -0.022 & $0.067^{* *}$ & 0.19 \\
R dPMC-SMAr & -0.041 & 0.042 & 0.16 \\
L OP4-R OP4 & $0.472^{* * *}$ & $0.322^{* * *}$ & -0.24 \\
L OP4-SMAr & $0.107^{* * *}$ & 0.025 & -0.16 \\
R OP4-SMAr & $0.154^{* * *}$ & $0.078^{* * *}$ & -0.17 \\
SMAr-L AIC & $0.289^{* * *}$ & $0.200^{* * *}$ & -0.14 \\
SMAr-R AIC & $0.314^{* * *}$ & $0.212^{* * *}$ & -0.16 \\
\hline
\end{tabular}

Mean $r_{\text {young }}$ and mean $r_{\text {elderly }}$ denote group-averaged functional connectivity (FC) in the 100 youngest and 100 oldest participants, respectively. $R$, right; L, left; $7 A$, superior parietal area 7A; OP4, area OP4 of the parietal operculum; SMAr, rostroventral supplementary motor area; $A I C$, anterior insular cortex; $d P M C$, dorsal premotor cortex.

${ }^{* *}$ significant at $p<0.001,{ }^{* * *}$ significant at $p<0.0001$.

Finally, even in simple tasks (i.e., auditory and visual choice reactions), age-related changes within the sensorimotor system appear to affect performance (Yordanova et al., 2004). Although area OP4 represents only a single node within the sensorimotor system, the altered task-independent coupling for this regions may indicate a neural correlate of the above-mentioned behavioral difficulties observed in older adults.

\section{Age-dependent differences in RS-FC between visual-attention and sensorimotor-related regions}

Age-related increases in RS-FC were observed for inter-system connections, i.e., between sensorimotor-related and visual attention-related regions (OP4-7A; see Figure 5). Importantly, RS-FC showed an anti-correlated pattern between those regions in the young subsample (Figure 4B). Thus, in young adults, task-independent neural activity in OP4 is accompanied by deactivation of area 7A and vice versa. This anti-correlation appears to be diminished in older adults, as RS-FC increased with age, approaching zero (see Figure 5). In other words, switching or mutual suppression (both of which would result in anticorrelation) between both sensorimotor and visual-attention network activity seems to be deteriorated in advanced age, potentially resulting in less distinct processing. This effect corroborates the fMRI study the functional seed regions were derived from (Roski et al., 2013). In that study we observed less differentiated taskdependent neural activity within areas 7A and OP4 in older adults, i.e., their activation patterns became less specific with age. Hence, the age-related changes of RS-FC observed here might reflect the previously demonstrated age-related alteration of a task-dependent activation pattern. Changes of intrinsic connectivity may hence be considered as a potential predictor for changes in task-dependent activation.

\section{Age-dependent differences in RS-FC between task-specific regions and task-general seeds}

Decreased RS-FC was observed between OP4 and the task-general SMAr, suggesting reduced communication between these regions with age. A similar effect was found for the attention-related area 7A. Here RS-FC was negative in the young subsample (see Table 3), the age-related FC increase (toward zero) reflects a loss of this anti-correlation, i.e., again a reduced communication. In other words, with age SMAr shows a less distinct connectivity with sensorimotor and visual attention related regions. In contrast, we found no significant age-related changes in RSFC between the second task-general region (i.e., AIC in each hemisphere), and the task-specific regions.

The AIC is known to be a highly integrative region with relevance for the processing of somatosensory, cognitive and socialemotional information (Kurth et al., 2010). Likewise, the SMA is known to integrate neural information relevant for the internal generation of movements (Picard and Strick, 1996; Jenkins et al., 2000; Thickbroom et al., 2000; Crosson et al., 2001; Weeks et al., 2001; Cunnington et al., 2002). In line with this, we here identified the task-general regions SMAr and AIC by significant task-based FC to all of the fMRI-based seeds. Hence, both regions seem to subserve higher-order cognitive processes interacting with both sensorimotor and visual attention specific regions. In their influential study, Dosenbach et al. (2006) proposed a "core system for the implementation of task sets." In that study, SMA and bilateral AIC were shown to be associated with the initiation and the maintenance of mental task sets. Notably, the present task-general regions closely correspond to this "core system" found by Dosenbach and colleagues. In another line of studies it was shown that regions in a very similar position as our task-general SMAr and AIC do not respond in a task-specific manner but rather to the degree of personal salience across tasks (e.g., Craig, 2002; Curtis and D'Esposito, 2003; Kerns et al., 2004). This "salience network" is thought to integrate highly processed sensory data with visceral, autonomic, and hedonic information (Damasio, 2000), so that the organism can decide what to do (or not to do) next. The reduced RS-FC with age between SMAr and the task-specific regions may hence imply difficulties for older adults to initiate, maintain, and switch activation in task-relevant functional systems. At the behavioral level, such difficulties were demonstrated for older adults during global task switching (Wasylyshyn et al., 2011) and dual-tasking (Verhaeghen et al., 2003; Just et al., 2008), that is, in situations where different mental task sets have to be constantly (re-)initiated or simultaneously maintained. The demonstrated age-related reduction in RS-FC between task-set control regions and task-specific regions (related to visual attention or sensorimotor processing) may reflect these difficulties at the neural level.

Moreover, our results also indicated a decreased functional coupling within the task-general network, i.e., between the SMAr, left and right AIC. As mentioned before, these regions represent basic nodes within a network assumed to be involved in taskswitching and dual-tasking processes. The reduced intercommunication of these regions in older adults strengthens our above interpretation that intrinsic age-related changes possibly mediate task-switching and dual-tasking difficulties in older adults. 
In line with this, increased task-switching costs were interpreted in terms of an age-related impairment in the ability to internally differentiate among task sets (Keith et al., 2004).

In summary, we found reduced task-independent communication within the task-general regions as well as between the task-general region SMAr and the functional seed regions. The reduced communication within the task-general regions may reflect difficulties for older adults during task-switching and dualtasking. Moreover, the reduced communication to the functional seed regions (sensorimotor and visual attention) may indicate more task specific impairments for older adults. In line with this, the interaction of the visual-attention and sensorimotor systems seems to be deteriorated in older adults (Szturm et al., 2013), possibly provoked by a deteriorated communication with the task-set system (task-general regions).

At the neural level, these intrinsic changes of intercommunication within and between the task-set system and functional seed regions might also explain the reduced distinctiveness of task-independent brain activity in the two functional systems (sensorimotor and visual attention), as found in our former study (Roski et al., 2013). In particular, the interplay of both systems may be controlled less precisely, potentially resulting in less distinct regional activations of task-specific brain regions.

Finally, an increased RS-FC with age was observed for the connection between the visual-attention-related APMC and the task-general SMAr. In contrast to the decreased communication between the attention-related area 7A and task-general SMAr, this age-related increase reflects an enhancement of communication for this connection. In line with this, it was shown that older (vs. younger) adults often recruit more frontal regions to successfully perform a visual attention task (Ansado et al., 2012; Li et al., 2013). Our findings of decreased FC between 7A and SMAr and increased FC between DPMC and SMAr thus reflects that the communication between the more posterior area 7A and SMAr declines with age, whereas the communication between the more anterior $\mathrm{APMC}$ and SMAr is enhanced. This effect may reflect an intrinsic posterior-to-anterior shift in aging (PASA; cf. Davis et al., 2008) for cognitive control within the visual attention system and, moreover, demonstrates that the PASA-effect, previously reported for regional brain activity, may also extend to RS-FC.

\section{LIMITATIONS AND FUTURE DIRECTIONS}

First, the current cross-sectional study offers the advantages of a large sample. However, some drawbacks have to be mentioned. Although a relation between age and changes in the interregional connection is clear, the causality between both is not positively determinable. In other words, there is no information on which variable caused the other. Moreover, we cannot completely exclude that additional variables, e.g., structural or neurochemical alterations, may influence the findings of the current study. In future research, the combination of structural MRI (e.g., diffusion tensor imaging), functional MRI, and neurochemical measurements, may extend the understanding of the causalities on FC changes in the aging brain. Second, since the analyzed functional networks reflect only parts of the visual-attention or sensorimotor system that showed an age-related loss of neural specificity, inferences from our results on age-related RS-FC changes between entire functional networks have to be regarded with caution. Nevertheless, our study indicates that healthy aging is associated with task-independent connectivity changes within and across task-specific brain network nodes. Furthermore, the current sample was derived by four different sites. Although all participants were screened for psychiatric and neurological disorders, the threshold for sub-clinical cognitive impairments may vary between them. Hence, the sample may contain some patients with sub-clinical symptoms. Finally, in the current sample we were unable to relate individual FC parameters to performance. Further studies should hence combine performance measurements with neuroimaging during task performance and task-free states in the same participants. This would permit investigating more direct relations of different age-related neural changes, their interdependencies, and their association with performance (see, e.g., Andrews-Hanna et al., 2007; Madden et al., 2010; Schulte et al., 2011).

\section{CONCLUSIONS}

The present study corroborates the notion that RS activity reflects the functional organization of the human brain (De Luca et al., 2006; Biswal et al., 2010), as RS activity in regions with similar functional characteristics was positively correlated, whereas RS activity in regions with different functional properties was negatively correlated but also revealed anti-correlation between task-specific and (co-activated) task general regions. Second, agerelated changes in network FC seem to be connection-specific, as not all RS connections were affected, and changes comprised both increases and decreases in RS-FC. Third, the majority of the observed age-related changes indicated a reduction of communication in the aging brain, as both correlations and anticorrelations were attenuated. Task-general regions, presumably relevant for the implementation and maintenance of task sets, showed reduced interregional RS-FC, in line with well-known difficulties of older adults in task-switching or dual-tasking. Furthermore, the communication between system-specific brain regions and the global task-set system seems to be intrinsically deteriorated, potentially leading to less differentiated regional brain activity during visual-attention and sensorimotor tasks, respectively (Roski et al., 2013). Finally, an age-related posteriorto-anterior shift was observed for the RS connectivity between areas of the visual attention system, in line with the PASA theory (Davis et al., 2008). In conclusion, our findings demonstrate that previously observed behavioral and functional brain activity changes concur with intrinsic FC changes in the healthy aging brain.

\section{ACKNOWLEDGMENTS}

Funding was granted by the National Institute of Mental Health (R01-MH074457; Peter T. Fox, Angela R. Laird, Simon B. Eickhoff), the Initiative and Networking Fund of the Helmholtz Association within the Helmholtz Alliance on Systems Biology (Human Brain Model; Karl Zilles, Simon B. Eickhoff), the Helmholtz Alliance for Mental Health in an Aging Society (HelMA; Katrin Amunts, Karl Zilles), and the German Research Foundation (DFG, EI 816/4-1, Simon B. Eickhoff; and LA 3071/31, Robert Langner, Simon B. Eickhoff). 


\section{SUPPLEMENTARY MATERIAL}

The Supplementary Material for this article can be found online at: http://www.frontiersin.org/journal/10.3389/ fnagi.2013.00067/abstract

Figure S1 | Visualization of the co-activation map for each task-specific seed region as revealed by meta-analytic connectivity modeling (MACM). L, left; R, right; DPMC, dorsal premotor cortex; 7A, superior parietal area 7A; OP4, area OP4 of the parietal operculum.

\section{REFERENCES}

Amiez, C., Kostopoulos, P., Champod, A. S., and Petrides, M. (2006). Local morphology predicts functional organization of the dorsal premotor region in the human brain. J. Neurosci. 26, 2724-2731. doi: 10.1523/JNEUROSCI.473905.2006

Andrews-Hanna, J. R., Snyder, A. Z., Vincent, J. L., Lustig, C., Head, D., Raichle, M. E., et al. (2007). Disruption of large-scale brain systems in advanced aging. Neuron 56, 924-935. doi: 10.1016/j.neuron.2007.10.038

Ansado, J., Monchi, O., Ennabil, N., Faure, S., and Joanette, Y. (2012). Loaddependent posterior-anterior shift in aging in complex visual selective attention situations. Brain Res. 1454, 14-22. doi: 10.1016/j.brainres.2012.02.061

Ashburner, J., and Friston, K. J. (2005). Unified segmentation. Neuroimage 26, 839-851. doi: 10.1016/j.neuroimage.2005.02.018

Baltes, P. B., and Lindenberger, U. (1997). Emergence of a powerful connection between sensory and cognitive functions across the adult life span: a new window to the study of cognitive aging? Psychol. Aging 12, 12-21. doi: 10.1037/0882-7974.12.1.12

Bandettini, P. A., and Bullmore, E. (2008). Endogenous oscillations and networks in functional magnetic resonance imaging. Hum. Brain Mapp. 29, 737-739. doi: 10.1002/hbm.20607

Biswal, B., Yetkin, F. Z., Haughton, V. M., and Hyde, J. S. (1995). Functional connectivity in the motor cortex of resting human brain using echo-planar MRI. Magn. Reson. Med. 34, 537-541. doi: 10.1002/mrm.1910340409

Biswal, B. B., Mennes, M., Zuo, X. N., Gohel, S., Kelly, C., Smith, S. M., et al. (2010). Toward discovery science of human brain function. Proc. Natl. Acad. Sci. U.S.A. 107, 4734-4739. doi: 10.1073/pnas.0911855107

Brown, M. R., Desouza, J. F., Goltz, H. C., Ford, K., Menon, R. S., Goodale, M. A., et al. (2004). Comparison of memory- and visually guided saccades using event-related fMRI. J. Neurophysiol. 91, 873-889. doi: 10.1152/jn.00382.2003

Buckner, R. L., and Carroll, D. C. (2007). Self-projection and the brain. Trends Cogn. Sci. 11, 49-57. doi: 10.1016/j.tics.2006.11.004

Craig, A. D. (2002). How do you feel? Interoception: the sense of the physiological condition of the body. Nat. Rev. Neurosci. 3, 655-666. doi: 10.1038/nrn894

Crosson, B., Sadek, J. R., Maron, L., Gokcay, D., Mohr, C. M., Auerbach, E. J., et al. (2001). Relative shift in activity from medial to lateral frontal cortex during internally versus externally guided word generation. J. Cogn. Neurosci. 13, 272-283. doi: 10.1162/089892901564225

Cunnington, R., Windischberger, C., Deecke, L., and Moser, E. (2002). The preparation and execution of self-initiated and externally-triggered movement: a study of event-related fMRI. Neuroimage 15, 373-385. doi: 10.1006/nimg.2001.0976

Curtis, C. E., and D'Esposito, M. (2003). Persistent activity in the prefrontal cortex during working memory. Trends Cogn. Sci. 7, 415-423. doi: 10.1016/S13646613(03)00197-9

Damasio, A. R. (2000). The Feeling of What Happens: Body and Emotion in the Making of Consciousness. Orlando, FL: Mariner Books.

Daselaar, S. M., Fleck, M. S., Dobbins, I. G., Madden, D. J., and Cabeza, R. (2006). Effects of healthy aging on hippocampal and rhinal memory functions: an event-related fMRI study. Cereb. Cortex 16, 1771-1782. doi: 10.1093/cercor/bhj112

Davis, S. W., Dennis, N. A., Daselaar, S. M., Fleck, M. S., and Cabeza, R. (2008). Que PASA? The posterior-anterior shift in aging. Cereb. Cortex 18, 1201-1209. doi: 10.1093/cercor/bhm155

De Luca, M., Beckmann, C. F., De Stefano, N., Matthews, P. M., and Smith, S. M. (2006). fMRI resting state networks define distinct modes of longdistance interactions in the human brain. Neuroimage 29, 1359-1367. doi: 10.1016/j.neuroimage.2005.08.035

Delbeuck, X., Van der Linden, M., and Collette, F. (2003). Alzheimer's disease as a disconnection syndrome? Neuropsychol. Rev. 13, 79-92. doi: 10.1023/A:1023832305702
Dennis, N. A., Hayes, S. M., Prince, S. E., Madden, D. J., Huettel, S. A., and Cabeza, R. (2008). Effects of aging on the neural correlates of successful item and source memory encoding. J. Exp. Psychol. Learn. Mem. Cogn. 34, 791-808. doi: 10.1037/0278-7393.34.4.791

Dosenbach, N. U. F., Visscher, K. M., Palmer, E. D., Miezin, F. M., Wenger, K. K., Kang, H. S. C., et al. (2006). A core system for the implementation of task sets. Neuron 50, 799-812. doi: 10.1016/j.neuron.2006.04.031

Eickhoff, S. B., Amunts, K., Mohlberg, H., and Zilles, K. (2006a). The human parietal operculum. II. Stereotaxic maps and correlation with functional imaging results. Cereb. Cortex 16, 268-279. doi: 10.1093/cercor/bhi106

Eickhoff, S. B., Schleicher, A., Zilles, K., and Amunts, K. (2006b). The human parietal operculum. I. Cytoarchitectonic mapping of subdivisions. Cereb. Cortex 16, 254-267. doi: 10.1093/cercor/bhil05

Eickhoff, S. B., and Grefkes, C. (2011). Approaches for the integrated analysis of structure, function and connectivity of the human brain. Clin. EEG Neurosci. 42, 107-121. doi: 10.1177/155005941104200211

Eickhoff, S. B., Jbabdi, S., Caspers, S., Laird, A. R., Fox, P. T., Zilles, K., et al. (2010). Anatomical and functional connectivity of cytoarchitectonic areas within the human parietal operculum. J. Neurosci. 30, 6409-6421. doi: 10.1523/JNEUROSCI.5664-09.2010

Eickhoff, S. B., Laird, A. R., Grefkes, C., Wang, L. E., Zilles, K., and Fox, P. T. (2009). Coordinate-based activation likelihood estimation meta-analysis of neuroimaging data: a random-effects approach based on empirical estimates of spatial uncertainty. Hum. Brain Mapp. 30, 2907-2926. doi: 10.1002/hbm.20718

Eyler, L. T., Sherzai, A., Kaup, A. R., and Jeste, D. V. (2011). A review of functional brain imaging correlates of successful cognitive aging. Biol. Psychiatry 70, 115-122. doi: 10.1016/j.biopsych.2010.12.032

Ford, K. A., Goltz, H. C., Brown, M. R., and Everling, S. (2005). Neural processes associated with antisaccade task performance investigated with event-related FMRI. J. Neurophysiol. 94, 429-440. doi: 10.1152/jn.00471.2004

Fox, M. D., and Raichle, M. E. (2007). Spontaneous fluctuations in brain activity observed with functional magnetic resonance imaging. Nat. Rev. Neurosci. 8, 700-711. doi: 10.1038/nrn2201

Fox, M. D., Zhang, D., Snyder, A. Z., and Raichle, M. E. (2009). The global signal and observed anticorrelated resting state brain networks. J. Neurophysiol. 101, 3270-3283. doi: 10.1152/jn.90777.2008

Fransson, P. (2005). Spontaneous low-frequency BOLD signal fluctuations: an fMRI investigation of the resting-state default mode of brain function hypothesis. Hum. Brain Mapp. 26, 15-29. doi: 10.1002/hbm.20113

Grady, C. (2012). The cognitive neuroscience of ageing. Nat. Rev. Neurosci. 13, 491-505. doi: 10.1038/nrn3256

Greicius, M. D., Krasnow, B., Reiss, A. L., and Menon, V. (2003). Functional connectivity in the resting brain: a network analysis of the default mode hypothesis. Proc. Natl. Acad. Sci. U.S.A. 100, 253-258. doi: 10.1073/pnas.0135058100

Halsband, U., and Lange, R. K. (2006). Motor learning in man: a review of functional and clinical studies. J. Physiol. Paris 99, 414-424. doi: 10.1016/j.jphysparis.2006.03.007

Hoffstaedter, F., Sarlon, J., Grefkes, C., and Eickhoff, S. B. (2012). Internally vs. externally triggered movements in patients with major depression. Behav. Brain Res. 228, 125-132. doi: 10.1016/j.bbr.2011.11.024

Inoue, K., Yamashita, T., Harada, T., and Nakamura, S. (2002). Role of human SII cortices in sensorimotor integration. Clin. Neurophysiol. 113, 1573-1578. doi: 10.1016/S1388-2457(02)00162-1

Jakobs, O., Langner, R., Caspers, S., Roski, C., Cieslik, E. C., Zilles, K., et al. (2012). Across-study and within-subject functional connectivity of a right temporo-parietal junction subregion involved in stimulus-context integration. Neuroimage 60, 2389-2398. doi: 10.1016/j.neuroimage.2012.02.037

Jenkins, I. H., Jahanshahi, M., Jueptner, M., Passingham, R. E., and Brooks, D. J. (2000). Self-initiated versus externally triggered movements. II. The effect of movement predictability on regional cerebral blood flow. Brain 123(Pt 6), 1216-1228. doi: 10.1093/brain/123.6.1216

Just, M. A., Keller, T. A., and Cynkar, J. (2008). A decrease in brain activation associated with driving when listening to someone speak. Brain Res. 1205, 70-80. doi: 10.1016/j.brainres.2007.12.075

Keith, C., Reddy, R. A., Hahn, H., Lang, H., and Tschierske, C. (2004). The carbosilane unit as a stable building block for liquid crystal design: a new class of ferroelectric switching banana-shaped mesogens. Chem. Commun. (Camb.), 1898-1899. doi: 10.1039/b407890j

Kerns, J. G., Cohen, J. D., Macdonald, A. W. 3rd., Cho, R. Y., Stenger, V. A., and Carter, C. S. (2004). Anterior cingulate conflict monitoring and adjustments in control. Science 303, 1023-1026. doi: 10.1126/science.1089910 
Kurth, F., Zilles, K., Fox, P. T., Laird, A. R., and Eickhoff, S. B. (2010). A link between the systems: functional differentiation and integration within the human insula revealed by meta-analysis. Brain Struct. Funct. 214, 519-534. doi: 10.1007/s00429-010-0255-Z

Laird, A. R., Eickhoff, S. B., Fox, P. M., Uecker, A. M., Ray, K. L., Saenz, J. J., et al. (2011). The BrainMap strategy for standardization, sharing, and meta-analysis of neuroimaging data. BMC Res. Notes 4:349. doi: 10.1186/1756-0500-4-349

Laird, A. R., Eickhoff, S. B., Kurth, F., Fox, P. M., Uecker, A. M., Turner, J. A., et al. (2009). ALE meta-analysis workflows via the brainmap database: progress towards a probabilistic functional brain atlas. Front. Neuroinform. 3:23. doi: 10.3389/neuro.11.023.2009

Li, L., Gratton, C., Fabiani, M., and Knight, R. T. (2013). Age-related frontoparietal changes during the control of bottom-up and top-down attention: an ERP study. Neurobiol. Aging 34, 477-488. doi: 10.1016/j.neurobiolaging.2012.02.025

Lindenberger, U., and Baltes, P. B. (1994). Sensory functioning and intelligence in old age: a strong connection. Psychol. Aging 9, 339-355. doi: 10.1037/08827974.9.3.339

Madden, D. J., Costello, M. C., Dennis, N. A., Davis, S. W., Shepler, A. M., Spaniol, J., et al. (2010). Adult age differences in functional connectivity during executive control. Neuroimage 52, 643-657. doi: 10.1016/j.neuroimage.2010.04.249

Nichols, T., Brett, M., Andersson, J., Wager, T., and Poline, J. B. (2005). Valid conjunction inference with the minimum statistic. Neuroimage 25, 653-660. doi: 10.1016/j.neuroimage.2004.12.005

O'sullivan, M., Jones, D. K., Summers, P. E., Morris, R. G., Williams, S. C., and Markus, H. S. (2001). Evidence for cortical "disconnection" as a mechanism of age-related cognitive decline. Neurology 57, 632-638. doi: 10.1212/WNL.57.4.632

Palomero-Gallagher, N., Mohlberg, H., Zilles, K., and Vogt, B. (2008). Cytology and receptor architecture of human anterior cingulate cortex. J. Comp. Neurol. 508, 906-926. doi: 10.1002/cne.21684

Palomero-Gallagher, N., Vogt, B. A., Schleicher, A., Mayberg, H. S., and Zilles, K. (2009). Receptor architecture of human cingulate cortex: evaluation of the four-region neurobiological model. Hum. Brain Mapp. 30, 2336-2355. doi: 10.1002/hbm. 20667

Picard, N., and Strick, P. L. (1996). Motor areas of the medial wall: a review of their location and functional activation. Cereb. Cortex 6, 342-353. doi: $10.1093 /$ cercor/6.3.342

Reetz, K., Dogan, I., Rolfs, A., Binkofski, F., Schulz, J. B., Laird, A. R., et al. (2012). Investigating function and connectivity of morphometric findingsexemplified on cerebellar atrophy in spinocerebellar ataxia 17 (SCA17). Neuroimage 62, 1354-1366. doi: 10.1016/j.neuroimage.2012.05.058

Reuter-Lorenz, P. A., and Park, D. C. (2010). Human neuroscience and the aging mind: a new look at old problems. J. Gerontol. B. Psychol. Sci. Soc. Sci. 65, 405-415. doi: 10.1093/geronb/gbq035

Rizzolatti, G., and Wolpert, D. M. (2005). Motor systems. Curr. Opin. Neurobiol. 15, 623-625. doi: 10.1016/j.conb.2005.10.018

Robinson, J. L., Laird, A. R., Glahn, D. C., Lovallo, W. R., and Fox, P. T. (2010). Metaanalytic connectivity modeling: delineating the functional connectivity of the human amygdala. Hum. Brain Mapp. 31, 173-184. doi: 10.1002/hbm.20854

Roski, C., Caspers, S., Lux, S., Hoffstaedter, F., Bergs, R., Amunts, K., et al. (2013). Activation shift in elderly subjects across functional systems: an fMRI study. Brain Struct. Funct. doi: 10.1007/s00429-013-0530-x. [Epub ahead of print].

Roskies, A. L., Schweitzer, N. J., and Saks, M. J. (2013). Neuroimages in court: less biasing than feared. Trends Cogn. Sci. 17, 99-101. doi: 10.1016/j.tics.2013.01.008

Salthouse, T. A. (2000). Aging and measures of processing speed. Biol. Psychol. 54, 35-54. doi: 10.1016/S0301-0511(00)00052-1

Satterthwaite, T. D., Elliott, M. A., Gerraty, R. T., Ruparel, K., Loughead, J., Calkins, M. E., et al. (2013). An improved framework for confound regression and filtering for control of motion artifact in the preprocessing of resting-state functional connectivity data. Neuroimage 64, 240-256. doi: 10.1016/j.neuroimage.2012.08.052

Schaefer, S., Huxhold, O., and Lindenberger, U. (2006). Healthy mind in healthy body? A review of sensorimotor-cognitive interdependencies in old age. Eur. Rev. Aging Phys. Act. 3, 45-54. doi: 10.1007/s11556-006-0007-5

Scheperjans, F., Eickhoff, S. B., Homke, L., Mohlberg, H., Hermann, K., Amunts, K., et al. (2008a). Probabilistic maps, morphometry, and variability of cytoarchitectonic areas in the human superior parietal cortex. Cereb. Cortex 18, 2141-2157. doi: $10.1093 /$ cercor/bhm 241
Scheperjans, F., Hermann, K., Eickhoff, S. B., Amunts, K., Schleicher, A., and Zilles, K. (2008b). Observer-independent cytoarchitectonic mapping of the human superior parietal cortex. Cereb. Cortex 18, 846-867. doi: 10.1093/cercor/bhm116

Schlegel, A., Alexander, P., Sinnott-Armstrong, W., Roskies, A., Tse, P. U., and Wheatley, T. (2013). Barking up the wrong free: readiness potentials reflect processes independent of conscious will. Exp. Brain Res. 229, 329-335. doi: 10.1007/ s00221-013-3479-3

Schulte, T., Muller-Oehring, E. M., Chanraud, S., Rosenbloom, M. J., Pfefferbaum, A., and Sullivan, E. V. (2011). Age-related reorganization of functional networks for successful conflict resolution: a combined functional and structural MRI study. Neurobiol. Aging 32, 2075-2090. doi: 10.1016/j.neurobiolaging.2009.12.002

Smith, S. M., Fox, P. T., Miller, K. L., Glahn, D. C., Fox, P. M., Mackay, C. E., et al. (2009). Correspondence of the brain's functional architecture during activation and rest. Proc. Natl. Acad. Sci. U.S.A. 106, 13040-13045. doi: 10.1073/pnas.0905267106

St Jacques, P. L., Dolcos, F., and Cabeza, R. (2009). Effects of aging on functional connectivity of the amygdala for subsequent memory of negative pictures: a network analysis of functional magnetic resonance imaging data. Psychol. Sci. 20, 74-84. doi: 10.1111/j.1467-9280.2008.02258.x

Szturm, T., Maharjan, P., Marotta, J. J., Shay, B., Shrestha, S., and Sakhalkar, V. (2013). The interacting effect of cognitive and motor task demands on performance of gait, balance and cognition in young adults. Gait Posture 38, 596-602. doi: 10.1016/j.gaitpost.2013.02.004

Thickbroom, G. W., Byrnes, M. L., Sacco, P., Ghosh, S., Morris, I. T., and Mastaglia, F. L. (2000). The role of the supplementary motor area in externally timed movement: the influence of predictability of movement timing. Brain Res. 874, 233-241. doi: 10.1016/S0006-8993(00)02588-9

Tomasi, D., and Volkow, N. D. (2012). Aging and functional brain networks. Mol. Psychiatry 17, 471, 549-458. doi: 10.1038/mp.2011.81

Verhaeghen, P., Steitz, D. W., Sliwinski, M. J., and Cerella, J. (2003). Aging and dual-task performance: a meta-analysis. Psychol. Aging 18, 443-460. doi: 10.1037/0882-7974.18.3.443

Wasaka, T., Nakata, H., Akatsuka, K., Kida, T., Inui, K., and Kakigi, R. (2005). Differential modulation in human primary and secondary somatosensory cortices during the preparatory period of self-initiated finger movement. Eur. J. Neurosci. 22, 1239-1247. doi: 10.1111/j.1460-9568.2005.04289.x

Wasylyshyn, C., Verhaeghen, P., and Sliwinski, M. J. (2011). Aging and task switching: a meta-analysis. Psychol. Aging 26, 15-20. doi: 10.1037/a0020912

Weeks, R. A., Honda, M., Catalan, M. J., and Hallett, M. (2001). Comparison of auditory, somatosensory, and visually instructed and internally generated finger movements: a PET study. Neuroimage 14, 219-230. doi: 10.1006/nimg.2001.0780

Yordanova, J., Kolev, V., Hohnsbein, J., and Falkenstein, M. (2004). Sensorimotor slowing with ageing is mediated by a functional dysregulation of motorgeneration processes: evidence from high-resolution event-related potentials. Brain 127, 351-362. doi: 10.1093/brain/awh042

Zu Eulenburg, P., Caspers, S., Roski, C., and Eickhoff, S. B. (2012). Meta-analytical definition and functional connectivity of the human vestibular cortex. Neuroimage 60, 162-169. doi: 10.1016/j.neuroimage.2011.12.032

Conflict of Interest Statement: The authors declare that the research was conducted in the absence of any commercial or financial relationships that could be construed as a potential conflict of interest.

Received: 12 August 2013; accepted: 11 October 2013; published online: 29 October 2013.

Citation: Roski C, Caspers S, Langner R, Laird AR, Fox PT, Zilles K, Amunts K and Eickhoff SB (2013) Adult age-dependent differences in resting-state connectivity within and between visual-attention and sensorimotor networks. Front. Aging Neurosci. 5:67. doi: 10.3389/fnagi.2013.00067

This article was submitted to the journal Frontiers in Aging Neuroscience.

Copyright (c) 2013 Roski, Caspers, Langner, Laird, Fox, Zilles, Amunts and Eickhoff. This is an open-access article distributed under the terms of the Creative Commons Attribution License (CC BY). The use, distribution or reproduction in other forums is permitted, provided the original author(s) or licensor are credited and that the original publication in this journal is cited, in accordance with accepted academic practice. No use, distribution or reproduction is permitted which does not comply with these terms. 